\title{
Proliferative Responses to Canine Thyroglobulin of Peripheral Blood Mononuclear Cells from Hypothyroid Dogs
}

\author{
Hiroyuki TANI ${ }^{1)}$, Tomoyo NABETANI ${ }^{1)}$, Kazumi SASAI ${ }^{1)}$ and Eiichiroh BABA ${ }^{1)}$ \\ ${ }^{1)}$ Department of Veterinary Internal Medicine, Division of Veterinary Science, Graduate School of Agriculture and Biological Sciences, \\ Osaka Prefecture University, 1-1 Gakuencho, Sakai, Osaka, 599-8531, Japan
}

(Received 26 July 2004/Accepted 12 November 2004) ABSTRACT. The immune responses of hypothyroid dogs to canine thyroglobulin (cTg) were evaluated for the proliferative ability of periph-
eral blood mononuclear cells (PBMC). PBMC from three hypothyroid dogs with high titers of thyroglobulin autoantibody (TgAA) and
3 clinically normal dogs were cultured with 5,10 , or $20 \mu \mathrm{g} / \mathrm{m} l$ of cTg for $72 \mathrm{hr}$. The proliferative responses of the cells were determined
by the level of incorporated BrdU. The numbers of cells expressing Thy- $1, \mathrm{CD} 4, \mathrm{CD} 8$ and IgG in the PBMC were counted by the immu-
nofluorescence method. Proliferative responses to cTg were observed in the cells from hypothyroid dogs. The number of cells express-
ing IgG and CD 8 in the hypothyroid dogs tended to be high compared with the clinically normal dogs. The CD $4^{+}$cells in cultures from
hypothyroid dogs increased depending upon the amount of cTg. There was a significant $(P<0.05)$ positive correlation between the num-
ber of CD $4^{+}$cells and the concentration of cTg in the cultures from hypothyroid dogs. These findings suggest a possible relationship
between canine hypothyroidism and cellular immunity. Loss of self tolerance to thyroid antigens in $\mathrm{CD} 4^{+} \mathrm{T}$ cells may play an important
role in the development of canine hypothyroidism.
KEY WORDS: canine, hypothyroidism, lymphocyte subpopulations, peripheral blood mononuclear cell, thyroglobulin.

J. Vet. Med. Sci. 67(4): 363-368, 2005

Hypothyroidism is a common endocrine disorder in dogs [6]. Thyroid hormone deficiency can cause a variety of clinical symptoms such as lethargy, obesity, skin diseases, cold intolerance, hair loss, and reproductive problems $[6,16]$. Most canine hypothyroidism results in the pathological process termed lymphocytic thyroiditis. Canine lymphocytic thyroiditis is considered to be an immune-mediated disease with clinical and histological similarities to Hashimoto's thyroiditis (HT) in man [3, 15]. HT is the thyroid autoimmune disease characterized by thyroid tissue destruction and inhibition of thyroid functions. Among thyroid antigens, thyroglobulin ( $\mathrm{Tg}$ ) and thyroperoxidase have been considered to be the major antigens related to HT. As antibody synthesis in autoimmune thyroid diseases (AITD) in humans includes IgG antibodies, which are helper $\mathrm{T}$ cell dependent, it is likely that helper T cell activity is important to the pathogenesis. The helper effect of $\mathrm{T}$ cells on antibody synthesis by $\mathrm{B}$ cells has been shown both in vivo and in vitro $[7,14,19]$.

Thyroglobulin-specific antibodies have also been found in a high proportion of clinically diagnosed hypothyroid dogs $[2,9,11,13]$. Thyroperoxidase antibodies were not found in one study [23], but uncharacterized microsomal antibodies have been reported in others [10, 15, 25]. The predominant thyroid antigen in dogs must be considered to be thyroglobulin. However, no evidence of a relationship between hypothyroidism and cellular immunity has been reported in dogs. With the aim of elucidating the role played by $T$ cells in canine hypothyroidism, we studied the proliferative responses of peripheral blood mononuclear cells (PBMC) to canine thyroglobulin.

\section{MATERIALS AND METHODS}

Animals: Three dogs with hypothyroidism were used (Table 1). Diagnosis of hypothyroidism was confirmed by clinical signs such as alopecia, lethargy, and weight gain as well as the following laboratory findings: hypercholesterolemia, low serum free thyroxine $\left(\mathrm{FT}_{4}\right)$ levels, high serum thyroid stimulating hormone (TSH) levels, and detectable thyroglobulin autoantibody (TgAA). The serum $\mathrm{FT}_{4}$ levels and TSH levels were determined by a commercial diagnostic laboratory (IDEXX Laboratories, KK, Tokyo, Japan). The detection method for canine TgAA in serum is described below. Two of three dogs (HYP-2, HYP-3) were treated with thyroxine replacement therapy for over one year. There was no change in treatment during the course of the study. Three purebred beagle dogs were also studied as normal controls (Table 1). They were determined as clinically normal by physical examination and laboratory data. The present study was conducted in accordance with NIH guidelines and the regulations of our Institutional Animal Care and Use Committee, and the dogs' owners signed a consent form before enrolling their dogs.

Thyroglobulin: cTg was purified from histologic normal thyroid glands from two clinically normal, purebred beagle dogs by gel filtration on a Sepharose CL-4B column $(2.5 \times$ $75 \mathrm{~cm}$, Amersham Pharmacia Biotech UK, Little Chalfont, Buckinghamshire, UK) as described in detail previously [22]. The purified cTg was characterized in our previous report [22]. The serum $\mathrm{FT}_{4}$ and $\mathrm{TSH}$ levels in the beagle dogs were normal. The serum was stored at $-80^{\circ} \mathrm{C}$ and used as normal controls.

ELISA for thyroglobulin autoantibody: Canine TgAA in the serum samples was detected by the method of Iversen et 
Table 1. Measurement of serum free $\mathrm{T}_{4}, \mathrm{TSH}$ and TgAA titer in hypothyroid dogs and normal dogs

\begin{tabular}{llcccccc}
\hline & Breed & Sex & $\begin{array}{c}\text { Age } \\
(\text { year })\end{array}$ & $\begin{array}{c}\text { Weight } \\
(\mathrm{kg})\end{array}$ & $\begin{array}{c}\mathrm{FT}_{4}{ }^{*} \\
(\mathrm{pmol} / l)\end{array}$ & $\begin{array}{c}\mathrm{TSH}^{*} \\
(\mathrm{ng} / \mathrm{m} l)\end{array}$ & $\begin{array}{c}\mathrm{TgAA}^{*} \\
\text { titer }\end{array}$ \\
\hline $\begin{array}{l}\text { Hypothyroid } \\
\text { HYP-1 }\end{array}$ & Beagle & $\mathrm{M}$ & 12 & 14.4 & 4.5 & 2.90 & $>2560$ \\
HYP-2 & Golden Retriever & $\mathrm{F}$ & 6 & 30.6 & $<3.0$ & 2.20 & $>2560$ \\
HYP-3 & Golden Retriever & $\mathrm{M}$ & 2 & 37.0 & $<3.0$ & 0.54 & $>2560$ \\
Normal & & & & & & & \\
Normal-1 & Beagle & $\mathrm{M}$ & 2 & 14.0 & 13.2 & 0.09 & $<20$ \\
Normal-2 & Beagle & $\mathrm{M}$ & 5 & 14.3 & 13.4 & 0.14 & $<20$ \\
Normal-3 & Beagle & $\mathrm{F}$ & 6 & 15.4 & 16.0 & 0.63 & $<20$ \\
\hline
\end{tabular}

* The data in the first medical examination before any treatments. F, Female; M, Male; $\mathrm{FT}_{4}$, Serum free thyroxine concentrations; TSH, Serum thyroid stimulating hormone concentrations; TgAA, Thyroglobulin autoantibody.

al. [13], with minor modifications [22]. Horseradish peroxidase-conjugated sheep anti-dog IgG (Serotec Ltd, Kidlington, Oxford, UK) was used. Serum samples were titrated in 2 fold dilutions from $1 / 20$ to $1 / 2,560$. The standard curve, negative for TgAA, was constructed from duplicate tests of pooled sera from two clinically normal dogs that were euthanatized for purification of thyroglobulin. Serum samples were judged to be positive at dilutions in which the optical density values exceeded twice the standard deviation above the negative curve. The intra-assay coefficient of variation in the present assay was less than $5 \%$. Each sample was assayed in duplicate in the same manner.

Proliferation assay and evaluation of lymphocyte subpopulations: Canine PBMC were evaluated for proliferative responses by the modified method of Wagner et al. [26]. Lymphocyte subpopulations were evaluated by counting the number of cells expressing Thy-1, CD4, CD8, or IgG in the PBMC. These tests were repeated twice or three times in each dog.

Ten milliliters of heparinized peripheral blood were collected from the cephalic vein, and diluted 1:1.4 with Hank's balanced salts solution (HBSS: Sigma Chemical, St. Louis, MO, U.S.A.). Lymphocyte Separation Solution ( $\mathrm{d}=1.077$, Nacalai Tesque, Inc., Kyoto, Japan) was used for separation of PBMC from whole blood. The mixture, 8 volumes of diluted blood in 3 volumes of Lymphocyte Separation Solution, was centrifuged at $400 \times \mathrm{g}$ for $30 \mathrm{~min}$ at room temperature. The PBMC at the interface were aspirated and washed twice with $5 \mathrm{~m} l$ of HBSS by centrifugation at $840 \times$ $\mathrm{g}$ for $5 \mathrm{~min}$ at $4^{\circ} \mathrm{C}$. After washing, the isolated cells were suspended in RPMI 1640 (SIGMA) supplemented with 10\% fetal calf serum, $100 \mathrm{U}$ penicillin/m $l$ and $100 \mu \mathrm{g}$ streptomy$\operatorname{cin} / \mathrm{ml}$. The number of isolated cells was quantified microscopically. Viability was determined by the trypan blue dye exclusion test.

One hundred microliters of each cell suspension with $5 \times$ $10^{5} \mathrm{cells} / \mathrm{m} l$ was added to wells of $96-$ well flat bottom tissue microculture plates (Iwaki, Tokyo, Japan). As an antigen, canine $\mathrm{Tg}$ diluted with culture medium at final concentrations of 5,10 , or $20 \mu \mathrm{g} / \mathrm{m} l$ was used. Concanavalin A (Con A; Wako Pure Chemical Industries, Ltd, Osaka, Japan) was also diluted with culture medium at the final concentration of $4 \mu \mathrm{g} / \mathrm{m} l$ as a mitogen. These antigens were sterilized by filtration with $0.22 \mu \mathrm{m}$ pore size membrane filter before adding to the cultures. One hundred microliters of the different concentrations of canine $\mathrm{Tg}$, Con A, or culture medium were added to the wells for a final volume of 200 $\mu l$. Each culture condition for each sample was tested in quadruplicate in the same culture. Two of the four wells were used for the proliferation assay, the rest of the wells were used for immunofluorescence. The cultures were incubated at $37^{\circ} \mathrm{C}$ with $5 \% \mathrm{CO}_{2}$ for $72 \mathrm{hr}$.

The BrdU assay procedure was carried out according to the original protocol from the manufacturer (Cell Proliferation ELISA BrdU, colorimetric; Roche Diagnostics, Mannheim, Germany). The reaction was quantified by measuring the optical density at a wavelength of $450 \mathrm{~nm}$ and a reference wavelength of $595 \mathrm{~nm}$. The stimulation index (SI) value (mean optical density values of antigen stimulated cultures/mean optical density values of medium-only cultures) was calculated for each culture treatment.

Immunofluorescence was used for evaluation of the lymphocyte subpopulations in PBMC. After incubation, the cells in each culture condition were recovered from each well of the microculture plates and washed twice with HBSS by centrifugation at $470 \times \mathrm{g}$ for $10 \mathrm{~min}$ at $4^{\circ} \mathrm{C}$. The cells were resuspended in HBSS and placed on a silanized slide (Dako Cytomation Co., Ltd., Glostrup, Denmark). The cell smears were dried and stored at $-80^{\circ} \mathrm{C}$ for the immunofluorescence assay. The smears were exposed to mouse anti-dog Thy-1, rat anti-dog CD4, rat anti-dog CD8 (Serotec) or FITC labeled goat anti-dog IgG (ICN/Cappel, Aurora, Ohio, U.S.A.) diluted at 1:50 with HBSS for $1 \mathrm{hr}$ at $37^{\circ} \mathrm{C}$. After three times washing with HBSS, the smears were subsequently exposed to FITC labeled goat anti-mouse IgG (Sigma) or FITC labeled goat anti-rat IgG (Leinco Technologies, Inc., Missouri, U.S.A.) diluted at 1:50 with HBSS for $1 \mathrm{hr}$ at $37^{\circ} \mathrm{C}$ except the slides for IgG detection. After three times washing with HBSS, the smears were counterstained with $0.1 \%$ Evans blue. The fluorescent signals were captured with a digital microscope camera (PDMC Ie: Polaroid Co., Waltham, MA, U.S.A.). The number of cells expressing Thy-1, CD4, CD8, and IgG was 

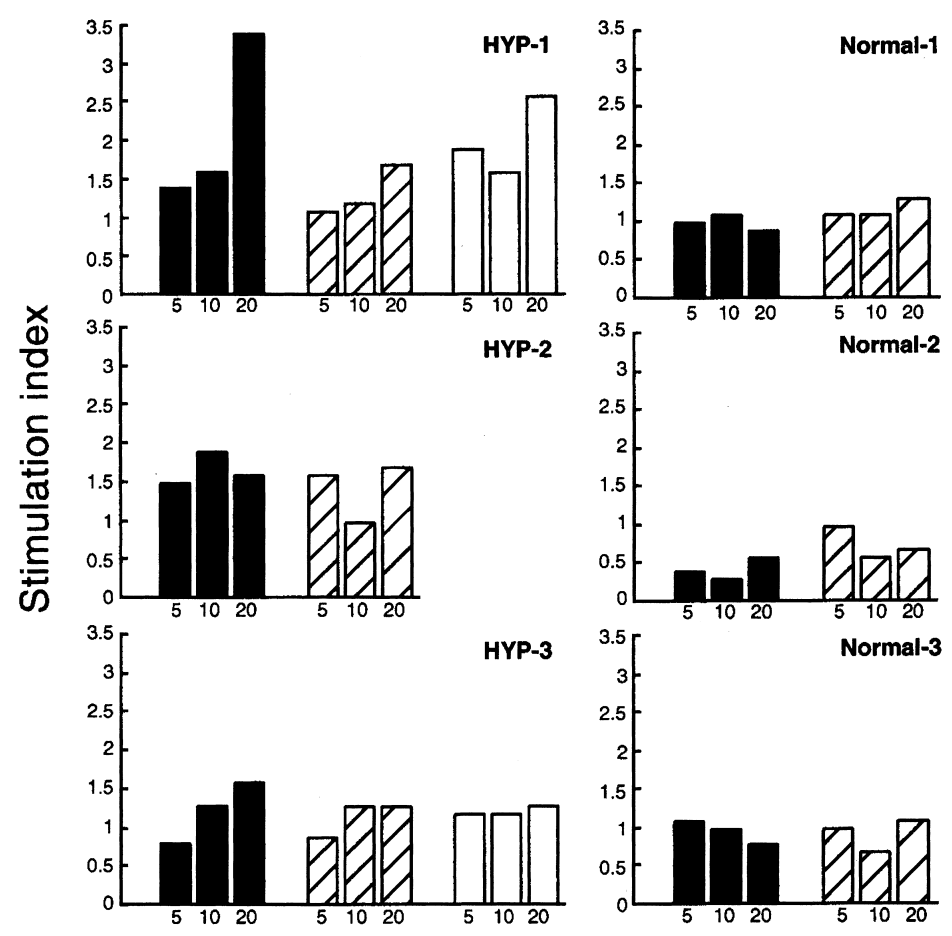

Tg concentration $(\mu \mathrm{g} / \mathrm{ml})$

Fig. 1. Mean proliferative responses from replicate assays of PBMC from hypothyroid dogs and normal dogs. The PBMC from hypothyroid dogs (HYP1, HYP-2 and HYP-3) and normal dogs (Normal-1, Normal-2 and Normal-3) were cultured with final concentrations of 5,10 , or $20 \mu \mathrm{g} / \mathrm{m} l$ of $\mathrm{cTg}$. The proliferative responses of the PBMC were determined by the level of incorporated BrdU as detailed in Materials and Methods. The results are expressed as the SI (mean of duplicate cultures). Bar graphs with different patterns denote the result of the first (black bar), second (shaded bar) and third (open bar) assays, respectively.

as percentages.

Statistical analysis: Mean values and standard deviations of the results in the each culture condition were calculated for mean values of the replications in each culture condition in each dog. Spearman's correlation coefficients were used for intercorrelations. A P value of less than 0.05 was considered statistically significant. The software used was Stat View (SAS institute Inc., North Carolina, U.S.A.).

\section{RESULTS}

Proliferative responses to $\mathrm{cTg}$ were observed in the PBMC from hypothyroid dogs (Fig. 1). The cells from two of three hypothyroid dogs (HYP-1 and HYP-2) showed highly proliferative responses to all concentrations of $\mathrm{cTg}$. The highest responses were observed in the cells from HYP1 in the presence of $20 \mu \mathrm{g} / \mathrm{m} l$ of cTg. The cells from one of the hypothyroid dogs (HYP-3) responded to 10 or $20 \mu \mathrm{g} / \mathrm{m} l$ of cTg, but did not react to $5 \mu \mathrm{g} / \mathrm{m} l$ of cTg in two of three tests. Mean values and standard deviations of the results from the proliferation assay are shown in Table 2. Dose-
Table 2. Proliferative responses of PBMC to Tg in hypothyroid dogs and normal dogs

\begin{tabular}{cccc}
\hline Antigen & $\begin{array}{c}\text { Concentration } \\
(\mu \mathrm{g} / \mathrm{m} l)\end{array}$ & \multicolumn{2}{c}{ Stimulationindex } \\
\cline { 3 - 4 } & 5 & $1.3 \pm 0.3$ & HYP $(\mathrm{n}=3)$ \\
$\mathrm{Tg}$ & 10 & $1.4 \pm 0.1$ & $0.9 \pm 0.2$ \\
& 20 & $1.9 \pm 0.6$ & $0.8 \pm 0.3$ \\
$\mathrm{ConA}$ & 4 & $18.5 \pm 7.6$ & $14.6 \pm 0.4$ \\
\hline
\end{tabular}

Values are expressed as the mean SI \pm SD of the mean values of replications in 3 dogs. Tg, Canine thyroglobulin; ConA, Concanavalin A; HYP, Hypothyroid dog; Normal, Normal dog.

dependence of the cTg-induced proliferation was not observed in the cells from hypothyroid dogs. All PBMC were viable, as shown by their proliferative responses to Con A.

There was a tendency toward a higher number of cells expressing IgG in cultures from the hypothyroid dogs compared to normal dogs (Table 3). The difference in the number of cells expressing Thy-1 was not observed between 
Table 3. Percentages of the number of cells expressing Thy-1 and IgG per five hundred of PBMC in the various culture conditions

\begin{tabular}{|c|c|c|c|c|c|}
\hline \multirow{3}{*}{ Antigen } & \multirow{3}{*}{$\begin{array}{l}\text { Concentration } \\
(\mu \mathrm{g} / \mathrm{m} l)\end{array}$} & \multicolumn{4}{|c|}{ Percentage of positive cells } \\
\hline & & \multicolumn{2}{|c|}{$\operatorname{HYP}(n=3)$} & \multicolumn{2}{|c|}{ Normal $(n=3)$} \\
\hline & & Thy-1 & IgG & Thy-1 & IgG \\
\hline Pre & & $60.1 \pm 5.4$ & $16.6 \pm 8.8$ & $57.8 \pm 5.6$ & $12.6 \pm 1.6$ \\
\hline \multirow[t]{4}{*}{$\mathrm{Tg}$} & 0 & $60.8 \pm 3.4$ & $20.7 \pm 10.7$ & $60.1 \pm 4.8$ & $13.3 \pm 3.1$ \\
\hline & 5 & $64.2 \pm 5.6$ & $21.9 \pm 7.2$ & $63.0 \pm 9.9$ & $13.3 \pm 2.9$ \\
\hline & 10 & $60.9 \pm 4.3$ & $22.5 \pm 12.6$ & $58.9 \pm 1.6$ & $13.7 \pm 2.2$ \\
\hline & 20 & $68.7 \pm 6.1$ & $21.4 \pm 9.1$ & $65.3 \pm 8.4$ & $14.2 \pm 2.4$ \\
\hline
\end{tabular}

Values are expressed as the mean \pm SD of the mean values of replications in 3 dogs. Pre, Pretreatment; Tg, Canine thyroglobulin; HYP, Hypothyroid dog; Normal, Normal dog.

Table 4. Percentages of the number of cells expressing CD4 and CD8 per five hundred of $\mathrm{PBMC}$ in the various culture conditions

\begin{tabular}{|c|c|c|c|c|c|}
\hline \multirow{3}{*}{ Antigen } & \multirow{3}{*}{$\begin{array}{l}\text { Concentration } \\
(\mu \mathrm{g} / \mathrm{m} l)\end{array}$} & \multicolumn{4}{|c|}{ Percentage of positive cells } \\
\hline & & \multicolumn{2}{|c|}{$\operatorname{HYP}(n=3)$} & \multicolumn{2}{|c|}{$\operatorname{Normal}(n=3)$} \\
\hline & & CD4+ & CD8+ & CD4+ & CD8+ \\
\hline Pre & & $28.5 \pm 3.0$ & $22.8 \pm 8.0$ & $29.0 \pm 3.4$ & $18.4 \pm 3.2$ \\
\hline \multirow[t]{4}{*}{$\operatorname{Tg}$} & 0 & $29.4 \pm 3.2$ & $23.1 \pm 6.2$ & $30.1 \pm 6.6$ & $19.4 \pm 3.1$ \\
\hline & 5 & $34.1 \pm 3.6$ & $23.7 \pm 8.3$ & $30.8 \pm 5.6$ & $17.8 \pm 2.3$ \\
\hline & 10 & $34.1 \pm 3.3$ & $22.8 \pm 8.9$ & $31.3 \pm 6.8$ & $20.3 \pm 3.4$ \\
\hline & 20 & $39.5 \pm 5.8$ & $27.3 \pm 8.3$ & $33.6 \pm 7.2$ & $18.0 \pm 6.0$ \\
\hline
\end{tabular}

Values are expressed as the mean $\pm \mathrm{SD}$ of the mean values of replications in 3 dogs. Pre, Pretreatment; Tg, Canine thyroglobulin; HYP, Hypothyroid dog; Normal, Normal dog.

hypothyroid dogs and normal dogs. The number of $\mathrm{CD}^{+}$ cells in cultures from hypothyroid dogs increased in the presence of $\mathrm{cTg}$ (Table 4$)$. There was a significant $(P<0.05)$ positive correlation between the number of $\mathrm{CD} 4^{+}$cells and the concentration of $\mathrm{cTg}$ existing in the cultures of cells from hypothyroid dogs. There was a tendency toward a higher number of $\mathrm{CD}^{+}$cells in cultures of cells from the hypothyroid dogs as compared with normal dogs.

\section{DISCUSSION}

Most studies on the pathogenesis of canine hypothyroidism have provided information on humoral immunity for development of diagnostic methods such as determination of serum TgAA, T3 autoantibody and T4 autoantibody levels [9]. The immunological and molecular pathogenesis of AITD in dogs has not been well characterized. The data reported here indicate that highly proliferative responses to cTg were present in the PBMC from hypothyroid dogs (Fig. 1 and Table 2). There was no such response in normal dogs. The results show the possibility that $\mathrm{cTg}$ is one of the major antigens of T cells in canine hypothyroidism. The subpopulation of the responding $\mathrm{T}$ cells may be mainly $\mathrm{CD} 4^{+}$cells. It must be indirect evidence of the reactivity of $\mathrm{CD}^{+}$cells that the number of $\mathrm{CD}^{+}$cells in the PBMC from hypothyroid dogs increased dependent upon the amount of $\mathrm{cTg}$ (Table 4). Additionally, the PBMC from hypothyroid dogs contained a higher number of cells expressing IgG as compared with normal dogs (Table 3). The observation also provided indirect evidence of continuous activation of $\mathrm{CD} 4^{+}$ cells in the PBMC from hypothyroid dogs. PBMC in the present study contained monocytes, which express $\mathrm{MHC}$ class II molecules as antigen presenting cells in the human and other species. Although the contribution of dog MHC subtypes to the disease in dogs are still under investigation [12], activated $\mathrm{CD}^{+}$cells may lead to the activation of $\mathrm{B}$ cells in hypothyroid dogs. Akasu et al. [1] have demonstrated that PBMC and CD8-depleted, CD4-enriched PBMC from patients with HT can proliferate in response to human $\mathrm{Tg}$ in vitro, and the responses are more apparent in CD8depleted, CD4-enriched PBMC. These responses were not observed in CD8-depleted, CD4-enriched PBMC from normal controls. They concluded that the function of suppressor $\mathrm{CD}^{+}$cells was partially disturbed in the AITD patients. Autoimmune thyroiditis that is induced in athymic mice can be transferred by $\mathrm{CD}^{+} \mathrm{T}$ cells, but a subfraction of normal $\mathrm{CD}^{+} \mathrm{T}$ cells prevented experimental autoimmune thyroiditis in mice, either directly or by inducing suppressor $\mathrm{T}$ cells [20]. The subpopulation of $\mathrm{CD}^{+} \mathrm{T}$ cells therefore has an important regulatory role in maintaining tolerance to $\mathrm{Tg}$ under normal circumstances [21]. Our results suggest that $\mathrm{CD}^{+} \mathrm{T}$ cells may play an essential role in the development of autoimmune thyroiditis in dogs as well as in human cases.

Our observations in this study provided results for only TgAA positive dogs. The proportion of hypothyroid dogs that have TgAA in serum is generally reported to be around $50 \%[2,9,10,24]$. In a recent study, canine TgAA titers were found to decline during L-thyroxine treatment [8]. 
Alternatively as proposed in a study with humans receiving L-thyroxine substitution therapy, there could be a reduction of antigenic substances through decreased stimulation of thyroid tissue by circulating TSH [18]. These findings suggest that complete disorder of thyroid functions attributable to long-lasting hypothyroidism may lead to a decline in antigenic substances resulting in reduced TgAA production. The reduction of the antigenic substances may also lead to a decrease in the reactivity of $\mathrm{CD} 4^{+} \mathrm{T}$ cells. The proliferative responses of PBMC to $\mathrm{cTg}$ also need to be evaluated in TgAA negative hypothyroid dogs.

In this study, we selected large dog breeds with hypothyroidism for repeated blood collections in large quantities. Therefore our results are not necessarily representative for all dog breeds with hypothyroidism. A variation in the prevalence of TgAA between different dogs breeds has been reported [9]. That finding suggests the heritability of lymphocytic thyroiditis. There may be a variation of pathologic differences in the disease among different dog breeds. A method for the proliferation assay using small quantities of blood must be developed for investigation of various dog breeds.

This is the first study to determine the reactivity of PBMC to canine Tg in hypothyroid dogs. The findings of the proliferation of the PBMC from hypothyroid dogs indicate a relationship between hypothyroidism and cellular immunity. Loss of self tolerance to thyroid antigens in $\mathrm{CD}^{+} \mathrm{T}$ cells may play an important role in the development of canine hypothyroidism. In the reports regarding the role of $\mathrm{T}$ cell epitopes of $\mathrm{Tg}$ in the pathogenesis of human AITD and experimental autoimmune thyroiditis in animal models, several pathogenic epitopes have been identified $[4,5,17]$. However, pathogenic $\mathrm{T}$ cell epitopes in canine AITD have not been reported, because the amino acid sequence of $\mathrm{cTg}$ has not been fully identified. Further investigations of the amino acid sequence of cTg may lead to a better understanding of the etiology of canine hypothyroidism.

ACKNOWLEDGMENTS. The authors are deeply grateful to Nestle Purina PetCare Company for partly supporting our study. This study was supported in part by a Grant-in Aid for Scientific Research No. 14760201 from the Japan Society for the Promotion of Science.

\section{REFERENCES}

1. Akasu, F., Morita, T., Resetkova, E., Yoshikawa, N., Carayon, P. and Volpe, R. 1993. Sensitization of T lymphocytes to thyroglobulin and thyroperoxidase in autoimmune thyroid diseases. Autoimmunity 14: 261-268.

2. Beale, K. M., Halliwell, R. E. W. and Chen, C. L. 1990. Prevalence of antithyroglobulin antibodies detected by enzymelinked immunosorbent assay of canine serum. J. Am. Vet. Med. Assoc. 196: 745-748.

3. Beierwalts, W. H. and Nishiyama, R. H. 1968. Dog thyroiditis: Occurence and similarity to Hashimoto's struma. Endocrinology 83: 501-508.

4. Carayanniotis, G. and Kong, Y. C. 2000. Pathogenic thyroglo- bulin peptides as model antigens: insights on the induction and maintenance of autoimmune thyroiditis. Int. Rev. Immunol. 19: $557-572$.

5. Carayanniotis, G. and Rao, V. P. 1997. Searching for pathogenic epitopes in thyroglobulin: parameters and caveats. Immunol. Today 18: 83-88.

6. Chastain, C. B. and Panciera, D. L. 1995. Hypothyroid diseases. pp. 1487-1500. In: Textbook of Veterinary Internal Medicine, 4th ed. (Ettinger, S. J. and Feldman, E.C. eds.), W.B. Saunders Company, Philadelphia.

7. Cong, Y. and Braley-Mullen, H. 1995. Proliferation and autoantibody production by mouse thyroglobulin (MTg)-specific B cells activated in vitro by MTg and MTg-specific T cells. Immunol. Lett. 45: 189-193.

8. Deeg, C. A., Kaspers, A., Hartman, K., Kraft, W. and Kaspers, B. 1997. Canine hypothyroidism: detection of anti-thyroglobulin antibodies. Tieäztl. Prax. 25: 170-173.

9. Graham, P. A., Nachreiner, R. F., Refsal, K. R. and Provencher-Bollinger, A. L. 2001. Lymphocytic thyroiditis. Vet. Clin. North Am. Small. 31: 915-933.

10. Haines, D. M., Lording, P. M. and Penhale, W. J. 1984. The detection of canine autoantibodies to thyroid antigens by enzyme-linked immunosorbent assay, hemagglutination and indirect immunofluorescence. Can. J. Comp. Med. 48: 262267.

11. Haines, D. M., Lording, P. M. and Penhale, W. J. 1984. Survey of thyroglobulin autoantibodies in dogs. Am. J. Vet. Res. 45: 1493-1497.

12. Happ, G. M. 1995. Thyroiditis - a model canine autoimmune disease. Adv. Vet. Sci. Comp. Med. 39: 97-139.

13. Iversen, L., Jensen, A. L., Hoier, R., Skydsgaard, M. and Kristensen, F. 1998. Development and validation of an improved enzyme-linked immunosorvent assay for the detection of thyroglobulin autoantibodies in canine serum samples. Domest. Anim. Endocrinol. 15: 525-536.

14. Janeway, C. A., Jr., Carding, S., et al. 1988. CD4 ${ }^{+} \mathrm{T}$ cells: specificity and function. Immunol. Rev. 101: 39-80.

15. Mizejewski, G. J., Baron, J. and Poissant, G. 1971. Immunologic investigations of naturally occurring canine thyroiditis. $J$. Immunol. 107: 1152-1160.

16. Panciera, D. L. 2001. Conditions associated with canine hypothyroidism. Vet. Clin. North Am. Small 31: 935-949.

17. Rao, V. P. and Carayanniotis, G. 1997. Contrasting immunopathogenic properties of highly homologous peptides from rat and human thyroglobulin. Immunology 90: 244-249.

18. Romaldini, J. H., Biancalana, M. M., Figueredo, D. I., Farah, C. S. and Mathias, P. C. 1996. Effects of L-thyroxine administration on antithyroid antibody levels, lipid profile, and thyroid volume in patients with Hashimoto's thyroiditis. Thyroid 6: 183-188.

19. Sanders, M. E., Makgoba, M. W. and Shaw, S. 1988. Human naive and memory $\mathrm{T}$ cells: reinterpretation of helper-inducer and suppressor-inducer subsets. Immunol. Today 9: 195-199.

20. Sugihara, S., Izumi, Y., et al. 1988. Autoimmune thyroiditis induced in mice depleted of particular T cell subsets. I. Requirement of Lyt-1 dull L3T4 bright normal T cells for the induction of thyroiditis. J. Immunol. 141: 105-113.

21. Taguchi, O. and Takahashi, T. 1996. Mouse models of autoimmune disease suggest that self-tolerance is maintained by unresponsive autoreactive T cells. Immunology 89: 13-19.

22. Tani, H., Shimizu, R., Sasai, K. and Baba, E. 2003. Recognition pattern of thyroglobulin autoantibody from hypothyroid dogs to tryptic peptides of canine thyroglobulin. J. Vet. Med. 
Sci. 65: 1049-1056.

23. Thacker, E. L., Davis, J. M., Refsal, K. R. and Bull, R. W. 1995. Isolation of thyroid peroxidase and lack of autoantibodies to the enzyme in dogs with autoimmune thyroid disease. Am. J. Vet. Res. 56: 34-38.

24. Thacker, E. L., Refsal, K. R. and Bull, R. W. 1992. Prevalence of autoantibodies to thyroglobulin, thyroxine, or triiodothyronine and relationship of autoantibodies and serum concentra- tion of iodothyronines in dogs. Am. J. Vet. Res. 53: 449-453.

25. Vajner, L. 1997. Lymphocytic thyroiditis in beagle dogs in a breeding colony: findings of serum autoantibodies. Vet. MedUS. 42: 333-338.

26. Wagner, U., Burkhardt, E. and Failing, K. 1999. Evaluation of canine lymphocyte proliferation: comparison of three different colorimetric methods with the $3 \mathrm{H}$-thymidine incorporation assay. Vet. Immunol. Immunopathol. 70: 151-159. 